\title{
Scientists
}

\section{Stressing the Importance of Development}

\author{
Tracy L. Bale \\ Associate professor of neuroscience; director, Neuroscience Center; University of Pennsylvania, Philadelphia, PA
}

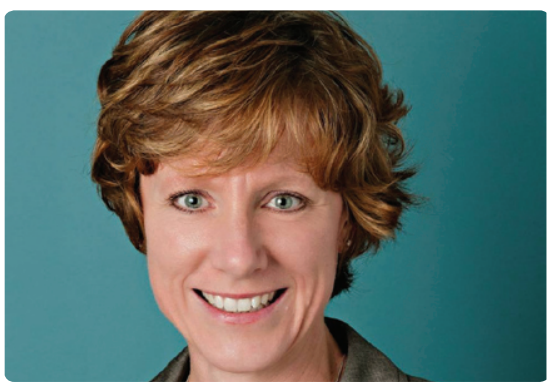

Tracy Bale's research into the influences of the prenatal environment on the developing brain caught our attention. Curious to know more, BioTechniques contacted her to find out about the ambition, character, and motivation that led to her success.

What interested you in looking at the effects of the prenatal environment?

In most neuropsychiatric diseases, symptoms almost always present after a stressful life event, even in individuals who are well medicated or who never presented with an affective disorder before. For example, a child with autism might be doing well, but if that child is disrupted or stressed by a schedule change, his symptoms may be exacerbated. This started me thinking about what could cause such drastic disruptions to stress pathways. Since it is unlikely that single genes cause the variance in stress response, I began looking at how they could develop. This led me to study the prenatal environment, specifically maternal stress and diet. We focus on how the prenatal environment and stress response during different periods of life can be a key predisposing factor toward disease. There are periods of vulnerability during prenatal development where changes in the mother's environment indirectly alter the programming of the fetal brain, and I have been working to define those specific time periods.

\section{What are you working on now?}

At the moment, we are trying to clarify the mechanisms by which most neuropsychiatric diseases have sex biases. There's a much higher presentation of autism and schizo- phrenia in males than in females, whereas depression and anxiety disorders occur more frequently in females. In addition, there are also sex differences in the effectiveness of treatment and the severity of symptoms. Our goal is to find out why it is that when we expose a mother to a given stressor during pregnancy, in spite of the fact that the rodents we are testing have both male and female offspring in the same uterus, the effect is sex specific.

We are also interested in the metabolic factors influencing obesity, another disorder with underlying stress components. We have ongoing studies looking at how maternal high-fat diets affect offspring, and have actually tracked an induced insensitivity to insulin, which is a precursor to diabetes, across multiple generations to see if the mom or dad can pass it on and if the male or female offspring inherit it. By looking across generations, we can begin to figure out how that programming occurs.

How do you experimentally approach these complex questions?

Once we identify a dam as pregnant, we assign her to either develop normally in her cage or to experience chronic stress during a very narrow time frame, the equivalent of the first trimester. We use one different mild stressor, which is psychological in nature, per day over the course of 7 days. So, she may have marbles put in her cage overnight that she will try to bury or avoid. Or maybe her bedding will be wet one day and the next day we will change her cage multiple times. The next day might include a short period of restraint. In the end, there is nothing that is painful or that changes her gestational length, litter size, or actual behavior. It's just enough to be upsetting to the mouse.

For our analysis, we look at different end points with a systems approach because behaviors are a culmination of many different factors. We use behavioral tests that have been validated with antidepressant and anxiolytic drugs to compare the control animals with the prenatally stressed animals. But behavioral testing can be confounded by many questions: Are the animals more stress sensitive or responsive? Are they differentially cognitive? Do they have better motor skills? So, we also examine physiological measures of stress hormones comparing the basal state with acute stress to monitor the animals' responses. And we can look at other physiological changes, including developmental differences, changes in gene expression profiles within particular areas of the brain, and proteomics studies on amniotic fluid or protein array comparisons of the placenta.

We work with mice, but obviously the goal is translational. So, studying placenta or amniotic fluid is advantageous since it is a lot easier to get those samples from humans than a part of the brain. Much of our current work is focused on identifying biomarkers for disease or altered stress response, which will also be translational.

\section{What is the most important open question in your field?}

The programming effects we see are typically epigenetic, so I think the most important question at the moment is how dynamic epigenetic marks really are and when they are vulnerable to change. Evidence suggests that for some genes methylation marks can be changed under stress or drug abuse. However, the problem is that we really don't have a way to test the relevance of those changes. We can measure whether or not a gene has increased or decreased methylation, but we can't test the importance of that particular level of methylation. This is something we still need to achieve.

Interviewed by Kristie Nybo, Ph.D. Image courtesy of Sheila Yamasaki. 䢐故

BioTechniques 51: 369 (December 2011)

doi 10.2144/000113744

To purchase reprints of this article, contact. biotechniques@fosterprinting.com 